We also agree that even when everything is functioning normally, computer workflow can sometimes lead to unexpected, and often surprising, situations. For example, Associate Editors of the ERJ must now assess the potential of all submitted papers for presentation as continuing medical education (CME)-accredited studies. In very simple terms, besides the three classical options, and their corresponding boxes indicating whether a paper should be accepted, revised or rejected, two new options have recently appeared on the ERJ Editors' dashboards asking them to indicate their choice about the CME qualifications of the manuscript in question. Failing (or forgetting) to indicate their choice by ticking the appropriate box blocks the system. One ERJ Associate Editor has recently pointed out to us that indicating whether a rejected paper should be considered for CME is certainly not logical as the answer to this question is obviously negative. Still, this Editor will have to tick one of the two available boxes for CME qualification, even when the choice applies to rejected papers (which will obviously not be qualified for CME as they have already been rejected). Failing to do so will prevent the Editor from validating his/her screen and finalising their decision.

This very simple example illustrates how big the gap can be between the human mind and computer software. The former can often be brilliantly mobile and flexible (a quality known by neuroscientists as "plasticity"), allowing clever deductions to be made and meaning unnecessary steps can be avoided. The latter is always heavily, and sometimes hopelessly, systematic and rigid (a property described by computer engineers as "reliability") to help their users avoid mistakes and oblivion. Human mood is rarely cold, being either fully overjoyed or exceedingly frustrated when experiencing successes or failures. The only heat we will ever feel from the computer might simply come from the overburdened electrical wires of a machine that has never been switched off. The list of contrasts between a scientist's mind and the computer system can be endless, and is certainly too long to be given in detail here. One thing we can almost be certain about relates to the sense of humour and humility that characterise mankind. We thank Prof. Quanjer for his sense of humour when sharing with us his challenging experience as a "retired junior" submitting author. As "unretired senior" scientists, we also feel frustrated seeing our young colleagues and our children working with computers much faster than we can do, but we are nonetheless grateful to these evil machines when we remember the amount of paperwork we did when submitting our manuscripts no longer than a decade ago. For the sake of our authors and Editorial Board, we humbly promise that we will try to improve the ERJ submission system to make life easier for all users, junior and senior colleagues alike.

However, we are not certain that computer machines will ever sense the joys or frustrations of their users. Quite honestly, we would be surprised and most worried if they do...

\section{A.T. Dinh-Xuan and V. Brusasco, ERJ Chief Editors on behalf of the ERJ Editorial Board}

Statement of Interest: Statements of interest for A.T. DinhXuan and V. Brusasco can be found at www.erj.ersjournals. $\mathrm{com} / \mathrm{misc} /$ statements.dtl

DOI: $10.1183 / 09031936.00004510$

\title{
Pulmonary hypertension and lung diseases: a suggestion for revision of the clinical classification
}

\section{To the Editors:}

We read with great interest the new clinical classification of pulmonary hypertension $(\mathrm{PH})[1]$, that represents a tremendous and successful effort to summarise the existing evidence of a rapidly evolving field in a physician-friendly manner. We want to focus on the inclusion of sarcoidosis, pulmonary Langerhans cells histiocytosis (PCLH) and lymphangioleiomyomatosis (LAM) in the subgroup of $\mathrm{PH}$ with unclear or multifactorial aetiologies (Group 5). Although raising such an issue might seem pedantic, we believe that there are good reasons for reconsidering the group place of these disorders.

Interstitial lung diseases (ILD) consist of disorders of known (e.g. collagen vascular disease, environmental, drug-induced) as well as unknown causes. The latter include idiopathic interstitial pneumonias, granulomatous lung disorders (e.g sarcoidosis), and other forms of ILDs, including LAM, PLCH and eosinophilic pneumonia [2].
Group 5.2 diseases may have a systemic component but their pulmonary manifestations far outnumber the manifestations of any other system. They mainly represent diffuse parenchymal lung diseases with distinctive and well-defined clinicopathological features that can affect all aspects of lung anatomy (interstitium, airspaces, peripheral airways and vessels), resulting in $\mathrm{PH}$ in a proportion of patients. Sarcoidosis presents with associated lung involvement in up to $95 \%$ of cases [3] and respiratory failure is the most common cause of death in Europe and the USA. Furthermore, mean pulmonary artery pressure has been significantly correlated with diffusion deficit and pulmonary vascular resistance with arterial oxygen tension in this setting [4]. PCLH is an ILD, preferentially affecting heavy smokers. LAM usually presents with cystic degeneration of the lungs and is often misdiagnosed initially as asthma or chronic obstructive pulmonary disease. It is a fact that the pathogenesis of $\mathrm{PH}$ for these disease entities is incompletely understood and may be multifactorial, but the same is true for all other ILDs [5]. Not surprisingly, no PAH-specific therapies have been approved for all these disease entities. 
Indeed, the current classification moves to the right direction by adopting a clinically meaningful way of thinking and avoiding unnecessary pathophysiological implications; $\mathrm{PH}$ owing to left heart disease (Group 2) and chronic thromboembolism (Group 4) are good examples. In this context, sarcoidosis, PLCH and LAM (Group 5.2) represent disorders that, according to the current evidence, are associated with $\mathrm{PH}$ mainly due to lung involvement and, therefore, we propose their inclusion in the third category ( $\mathrm{PH}$ owing to lung disease and/or hypoxia) in any future classification scheme.

\section{Tsangaris*, S. Orfanos* and D. Bouros"}

2nd Critical Care Dept, Attikon University Hospital, Athens, and "Dept of Pneumonology, Medical School, Democritus University of Thrace, Komotini, Greece.

Correspondence: I. Tsangaris, 2nd Dept of Critical Care Medicine, University Hospital Attikon, Medical School, University of Athens, 1 Rimini Str, Athens 12462, Greece, E-mail: itsagkaris@med.uoa.gr

\section{REFERENCES}

1 Galiè N, Hoeper MM, Humbert M, et al. Guidelines for the diagnosis and treatment of pulmonary hypertension. The Task Force for the Diagnosis and Treatment of Pulmonary Hypertension of the European Society of Cardiology (ESC) and the European Respiratory Society (ERS), endorsed by the International Society of Heart and Lung Transplantation (ISHLT). Eur Respir J 2009; 34: 1219-1263.

2 Travis WD, King TE, Bateman ED, et al. ATS/ERS international multidisciplinary consensus classification of idiopathic interstitial pneumonias. General principles and recommendations. Am J Respir Crit Care Med 2002; 165: 277-304.

3 Baughman RP, Teirstein AS, Judson MA, et al. Case Control Etiologic Study of Sarcoidosis (ACCESS) research group. Clinical characteristics of patients in a case control study of sarcoidosis. Am J Respir Crit Care Med 2001; 164: 1885-1889.

4 Nunes H, Humbert M, Capron F, et al. Pulmonary hypertension associated with sarcoidosis: mechanisms, haemodynamics and prognosis. Thorax 2006; 61: 68-74.

5 Behr J, Ryu JH. Pulmonary hypertension in interstitial lung disease. Eur Respir J 2008; 31: 1357-1367.

\section{Predicting persistence of wheezing: one algorithm does not fit all}

\section{From the authors:}

ScOTT et al. [1] have commented, in the light of outcomes of their "Isle of Wight Birth Cohort study" (IoWBC) [2, 3], on our findings on the risk factors for asthma in the Multicentre Allergy Study (MAS) birth cohort study [4]. We agree that the two studies lead to similar conclusions in regard to early origins of persistent wheezing, heterogeneity of childhood wheezing and the relevance of an atopic background. In addition, we followed their suggestion to build a prognostic score in accordance with the IoWBC [3] and to test its performance in the MAS database. Here we report the results of our analysis.

The IWBC prognostic algorithm comprises four risk factors: a family history of asthma; recurrent chest infections in infancy; absence of nasal symptoms at age $1 \mathrm{yr}$; and atopic sensitisation at age 4 yrs [3]. To make the data of the two cohorts comparable, we reduced our longitudinal study with followups every year to the four time points of the Isle of Wight Study: age 1, 2, 4 and 10 yrs. Due to missing values, this reduction resulted in a fairly smaller population sample $(n=765)$; therefore we increased the older age group by 8 and 9 yr olds and obtained a proportion of examined children similar to the one obtained in the IoWBC. The parameters we used were quite similar, although not identical, to those used in the IoWBC study (table 1). The frequency distribution of the risk score in the MAS cohort was similar to the one observed in the IoWBC; however, only three children with early wheezing had a risk score of 4 (table 2). A cut-off to predict persistent wheeze in the MAS cohort was therefore placed at a risk score of $\geqslant 3$. The positive predictive value (PPV) we obtained with this cut-off was 0.65 (95\% CI 0.44-0.82) (table 3). Interestingly, when adopting a cut-off of 3 , the PPV in the IoWBC study was 0.68 , i.e. almost the same as the one obtained in the MAS cohort (table 3) [3].

On this basis we may conclude, as proposed by ScOTT et al. [1], that the IoWBC score can be a starting point in developing a clinically relevant predictive tool. Preventive measures should not be advised to children whose wheezing is likely to undergo spontaneous remission; therefore, a high PPV is desirable. On this basis, we feel that an algorithm based on early wheezing, sensitisation to mites and elevated allergen exposure, might be also clinically useful because it resulted in a better prediction in the MAS cohort (PPV 0.83). On the other hand, a third algorithm, based on the presence of atopic eczema, immunoglobulin (Ig)E sensitisation to food allergens and specific polymorphisms of the filaggrin gene, was able to predict another subset of asthmatic children of the MAS cohort with a PPV and equal to 1.00 (95\% CI 65-100) [5]. Given the great heterogeneity of mechanisms and risk factors for wheezing disorders, multiple algorithms are likely necessary to predict, with enough confidence, persistence of wheezing in the children with early wheezing. 